\title{
MATHEMATICAL MODEL OF WELDING CIRCUIT IN ROBOTIC CONSUMABLE ELECTRODE ARC WELDING
}

\author{
G.A. TSYBULKIN \\ E.O. Paton Electric Welding Institute, NASU \\ 11 Kazimir Malevich Str., 03680, Kiev, Ukraine. E-mail: office@paton.kiev.ua
}

\begin{abstract}
In the article the mathematical model of dynamic processes, running in the welding circuit during robotic gas-shielded arc welding using consumable electrode, is investigated The welding circuit is considered as a self-stabilizing system with a feedback by the electrode melting rate. Unlike the known mathematical models, in the investigated model the dependence of the electrode melting rate on actual values of welding current and arc voltage is taken into account. The basic aim of the article is the study of reaction of welding circuit on external disturbances arising in the process of arc welding. In the frames of the developed model the criteria for asymptotic stability were established, which in addition to the already known criteria impose certain limits on the parameters of arc welding mode itself, which is important from the practical point of view. Using the computer modeling a good correlation of this model with the real processes, running in welding circuit, was illustrated. 14 Ref., 4 Figures.
\end{abstract}

Keywords : robotic arc welding, consumable electrode, welding circuit, mathematical model, stability of welding processes

To develop the effective algorithms for adaptive control of robotic consumable electrode arc welding, it is necessary to dispose, if possible, an accurate and, at the same time, rather simple mathematical model of dynamic processes, running in welding circuit. As one of such models the following system of equations is used $[1,2]$ :

$$
\left.\begin{array}{c}
\left(L_{1}+L_{2}\right) \frac{d i}{d t}+\left(R_{1}+R_{2}\right) i=u_{x}-u_{a}(i, l), \\
u_{a}(i, l)=u_{0}+E l+S_{a} i, \\
l=H-h, \\
h=h_{0}+\int_{0}^{t}\left(v_{e}-v_{m}\right) d t, \\
v_{m}=M i .
\end{array}\right\}
$$

In these equations and in Figure $1 i=i(t)$ is the welding current; $L_{1}, R_{1}, u_{x}, u_{s}$ is the inductance, internal resistance, open-circuit voltage and the voltage at the output terminals of the welding power source (WPC), respectively; $L_{2}$ is the inductance of the circuit formed by connecting wires; $R_{2}$ is the total resistance of electrode stickout, connecting wires, sliding contact in the torch nozzle and a part of the workpiece being welded; $u_{a}=u_{a}(l, i)$ is the arc voltage; $u_{0}$ is the sum of near-electrode voltage drops; $l=l(t)$ is the arc length; $E=\partial u_{d} / \partial l$ is the intensity of electric field in the arc column; $S_{a}=$ $=\partial u_{a} / \partial i$ is the tangent of the inclination angle of static volt-ampere characteristic of the arc in the vicinity of the working point of welding; $H=$ const is the distance between the end of the current-carrying nozzle and a free surface of welding pool; $h_{0}, h=h(t)$ is the initial and actual values of electrode stickout; $v_{e}=$ const, $v_{m}=$ $=v_{m}(t)$ are the speeds of feed and melting of electrode, respectively; $M=\partial v_{m} / \partial i$ is the steepness of current characteristic of electrode melting; $t$ is the actual time.

The mathematical model (1) is successfully used in solving the problems of geometric adaptation, i.e.

(c) G.A. TSYBULKIN, 2016 adaptation of welding automatic machine or robot to unexpected deviations of a welding tool from the axial line of joint being welded directly in the process of arc welding [2-4]. Meanwhile, it still remains unclear how effective the use of this model will be in solving other types of problems, in particular, the problem of adaptive control of arc welding modes themselves. The matter is that the dependence of the electrode melting rate $v_{m}(t)$ on the actual value of arc voltage $u_{a}(t)$ is not taken into account in the model (1). The main argument in favor of such simplification was the fact that the distance $H$ between the end of the torch and the workpiece being welded during robotic arc welding is maintained constant unlike in the manual arc welding. It was considered that in this case the voltage $u_{a}(t)$ also negligibly changes and, thus, has a low influence on melting rate of the electrode. Therefore, the calculated average value $u_{a}$ was actually included into the generalized parameter $M=$ const, namely which appeared in the last equation of the system (1). We should note that instead of the equality $v_{m}=M i$ sometimes (at high welding currents) the ratio $v_{m}^{m}=M i+N h i^{2}$ is used, where $N=$ const [3-6]. But also in this ratio the actual value of arc voltage $u_{a}(t)$ is not taken into account.

The electrode melting rate $v_{m}(t)$, as is known [7, 8], is proportional to the electrical power supplied to the arc gap, i.e. proportional to the product $u_{a}(t)$ by $i(t)$. On the other hand, due to the famous effect of arc self-regulation, the speed $v_{m}(t)$ in the steady mode is equal to the electrode feed speed $v_{e}$, i.e. $v_{m}(t)=v_{e}=$ $=$ const. Therefore, any changes of $u_{a}(t)$ will certainly result in the corresponding changes in $i(t)$. Taking this into account, let us specify the mathematical model (1) introducing the more correct ratio instead of the approximate equality $v_{m}=M i$

$$
v_{m}=K u_{a} i \text {. }
$$


In this ratio the constant coefficient $K$ includes electrical, thermophysical and geometric characteristics of consumable electrode. It can be easily calculated or experimentally determined if these characteristics are known. Furthermore, using the coefficient $K$ it is simply enough to consider that part of electrical power which is directly consumed for melting the electrode.

Excluding the variables $u_{a}, h, v_{m}$ from the new system of equations (i.e., from the system (1) taking into account the equality (2)), let us reduce it to two differential equations relative to the variables $i$ and $l$ :

$$
\left.\begin{array}{c}
L \frac{d i}{d t}=-R_{w} i-E l+u_{x}-u_{0}, \\
\frac{d l}{d t}=K\left(u_{0} i+E l i+S_{a} i^{2}\right)-v_{e},
\end{array}\right\}
$$

where

$$
L=L_{1}+L_{2}, R_{w}+R_{1}+R_{2}+S_{a} .
$$

It is easy to see, that the system of equations (3) is non-linear. It is important to check the conditions under which the dynamic processes described by these equations will bear a stable nature.

Conditions for welding process stability. Let us find the relations between the parameters of welding circuit and arc welding mode, guaranteeing steady (stationary) modes. For this purpose, let us introduce the constant values $i_{\infty}$ and $l_{\infty}$ in the equations (3) instead of the variables $i$ and $l$. As a result, we obtain the following equations

$$
\left.\begin{array}{c}
-R_{w \infty} i-E l_{\infty}+u_{x}-u_{0}=0, \\
K\left(u_{0} i_{\infty}+E l_{\infty} i_{\infty}+S_{a \infty} i^{2}\right)-v_{e}=0,
\end{array}\right\}
$$

from which we find:

$$
\begin{aligned}
& l_{\infty}=\frac{u_{x}-u_{0}}{E}-\frac{R_{w}}{E} i_{\infty}, \\
& R_{+\infty} i^{2}-u_{\chi \infty} i_{\infty}+\frac{v_{e}}{K}=0,
\end{aligned}
$$

where

$$
R_{+}=R_{w}+S_{a}=R_{1}+R_{2} .
$$

It is easy to check that fulfilling the condition

$$
v_{e}<\frac{K u_{x}^{2}}{4 R_{+}}
$$

the roots of the quadratic equation (7) are real and positive. Hence, if the inequality (9) is true and the limit $0<l(t)<l_{k}$ is provided, where $l_{k}$ is the critical value of arc length, at which its break occurs, then stationary modes of arc welding do certainly exist.

Let us replace the variables $i$ and $l$ in the differential equations (3) by the sums $i=i_{\infty}+\xi$ and $l=l_{\infty}+\lambda$ where $\xi$ and $\lambda$ are the deviations of actual values of welding current $i$ and arc length $l$ from the corresponding established values $i_{\infty}=\lim _{t \rightarrow \infty} i(t)$ and $l_{\infty}=\lim _{t \rightarrow \infty} l(t)$. Restricting by the terms containing variables $\stackrel{t \rightarrow \infty}{\xi}$ and $\lambda$ in the first degree and taking into account the equations (5), we shall obtain the system of differential equations of the first approximation:

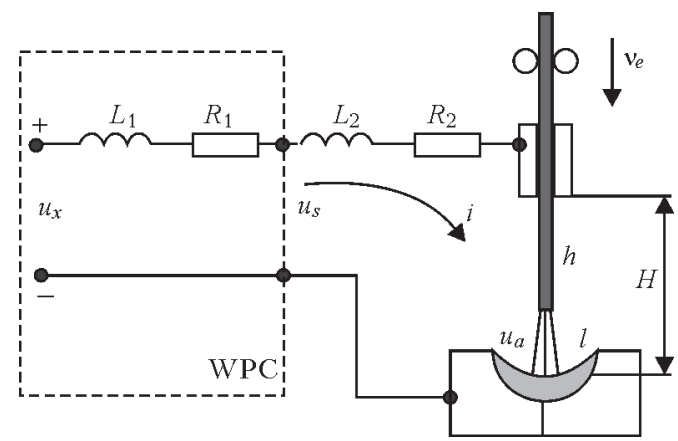

Figure 1. Scheme of welding circuit

$$
\left.\begin{array}{c}
L \frac{d \xi}{d t}=-R_{w} \xi-E \lambda, \\
\left.\frac{d \lambda}{d t}=K\left[u_{x}-\left(R_{w}-2 S_{a}\right) i_{\infty}\right)\right] \xi+K E i_{\infty} \lambda .
\end{array}\right\}
$$

Excluding the variable $\lambda$ from this system, we shall come to the only differential equation with respect to $\xi$ :

$$
\begin{gathered}
L \frac{d^{2} \xi}{d t^{2}}+\left(R_{w}-K E L i_{\infty}\right) \frac{d \xi}{d t}+ \\
+K E\left(u_{x}-2 R_{+\infty} i_{\infty}\right) \xi=0 .
\end{gathered}
$$

Thus, the problem concerning the stability of arc welding mode is reduced to investigation of stability of zero (trivial) solution $\xi=0$.

It is known [9] that for stability of the process, described by an ordinary differential equation of second order, it is necessary and sufficient that all its coefficients were positive (Stodola criterion). Hence, the variable $\xi=i-i_{\infty}$ in the equation (10) at $t \rightarrow \infty$ will tend to zero, if the following inequalities are correct:

$$
R_{w}>0, R_{w}-K E L i_{\infty}>0, u_{x}-2 R_{+} i_{\infty}>0 .
$$

The first inequality in (11) means that the algebraic sum $R_{w}=R_{1}+R_{2}+S_{a}$ should be positive. It is a known criterion of Kaufman-Nikitin [10-14]. In fact, the resistance $R_{1}$, included into this sum, is the absolute value of the angular coefficient of the volt-ampere characteristic of WPC. Hence, $R_{1}=\left|-\partial u_{s} / \partial i\right|>0$. The angular coefficient $S_{a}=\partial u_{a} / \partial i$, as is known [7, 8], can be both positive and negative. If $S_{a} \geq 0$, then $R_{w}>0$ also. Whereas if $S_{a}<0$, then to fulfill the condition $R_{w}>0$, it is necessary that according to (4) and (8) we obtained the inequality

$$
R_{+}-\left|S_{q}\right|>0 .
$$

The second and the third conditions in (11) impose limits on the inductance $L$ and on the relation between the parameters of arc welding mode $u_{x}$ and $i_{\infty}$. Let us write these conditions in the following way:

$$
L<\frac{R_{w}}{K E i_{\infty}}, \frac{u_{x}}{i_{\infty}}>2 R_{+} .
$$

Thus, in order that the process, described by the equation of the first approximation (10), was asymptotically stable, it is necessary and sufficient that the following conditions (9), (12), (13) were fulfilled. If the mentioned conditions are fulfilled and the disturbing effects are sufficiently low, then, according to the known Lyapunov theorem [9], the process, described by the initial non-linear equations (3) was also asymptotically stable. 
Reaction of welding circuit on external effects. The welding circuit including electric arc, consumable electrode supplied in the welding process at a certain speed $v_{e}$ and the welding power source WPC, from the point of view of the theory of automatic control represents, as is known [2, 7], a closed system with the internal negative feedback by the electrode melting rate. Thanks to this feedback, the system itself (without special devices) performs stabilization of the electrode melting rate $v_{m}$ at the level of the preset electrode feed speed $v_{e}$.

It is used to judge about the stabilizing properties of welding circuit, including quickness and accuracy of practicing the input actions, by its reaction to these influences. Studying the reaction of the mathematical model (3) on the similar effects, it is possible to evaluate the degree of its conformity (adequacy) to the real processes running in welding circuit, and, in particular, to compare the models (3) and (1).

For such kind of verification of the model (3) let us carry out the simulation experiment on the computer. Let us suppose that it is necessary to perform shielded-gas arc welding using consumable electrode with the diameter equal to $1 \mathrm{~mm}$. The welding current $i_{\infty} \approx 150 \mathrm{~A}$, and the arc length $l_{\infty} \approx 4 \mathrm{~mm}$. The values of welding circuit parameters are the following: $L=4 \cdot 10^{-4} \mathrm{H}, R_{1}=0.025 \mathrm{Ohm}, R_{2}=0.01 \mathrm{Ohm}, H=$

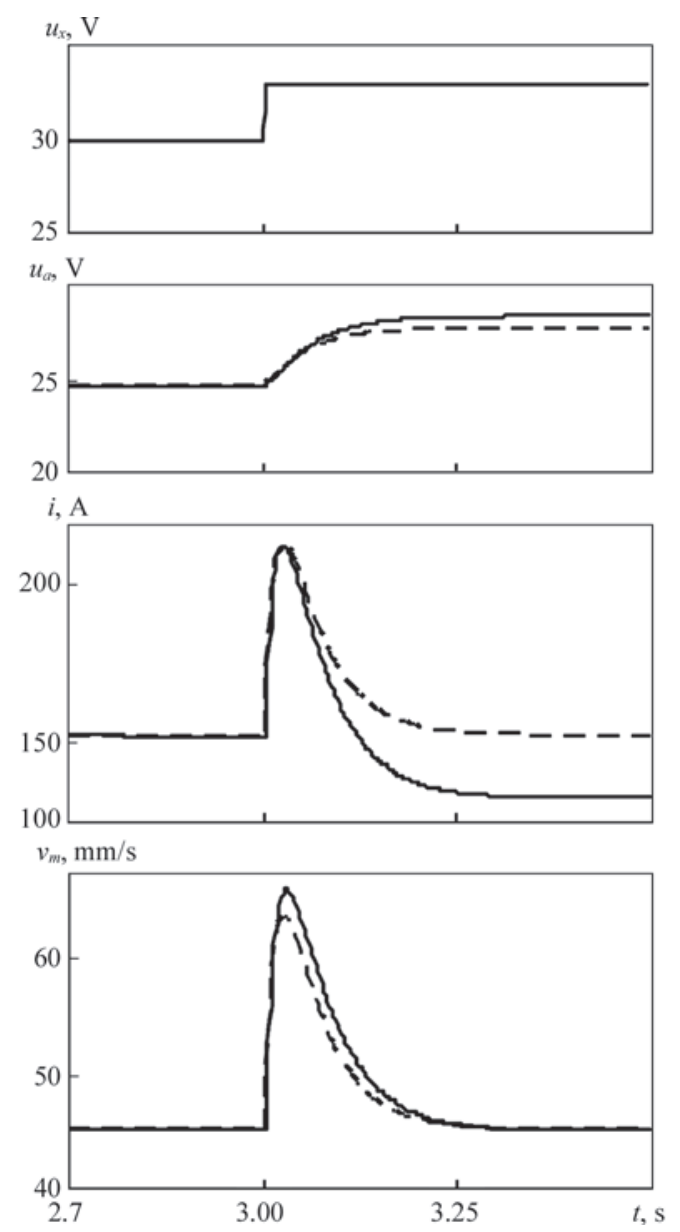

Figure 2. Reaction of welding circuit on disturbance $\Delta u_{x}=3 \mathrm{~V}$
$=17 \mathrm{~mm}, u_{0}=16 \mathrm{~V}, E=2 \mathrm{~V} / \mathrm{mm}, S_{a}=0.005 \mathrm{~V} / \mathrm{A}, K=$ $=0.012 \mathrm{~mm} /(\mathrm{A} \cdot \mathrm{V} \cdot \mathrm{s})$.

The voltage $u_{x}$ can be calculated using the equation (6): $u_{x}=E l_{\infty}+u_{0}{ }^{x}+R_{w} i_{\infty}=2 \cdot 4+16+0.04 \cdot 150=30 \mathrm{~V}$. Let us determine the electrode feed speed $v_{e}$ from the equation (7): $v_{e}=K i_{\infty}\left(u_{x}-R_{+} i_{\infty}\right)=0.012 \cdot 150 \cdot(30-$ $0.035 \cdot 150) \approx 45^{e} \mathrm{~mm} / \mathrm{s}$.

Substituting these values to the expressions (8), (12) and (13)

$$
\begin{gathered}
v_{e}<\frac{0,012 \cdot 30^{2}}{4 \cdot 0.035} \approx 77 \mathrm{~mm} / \mathrm{s}, \\
R_{+}-\left|S_{a}\right|=0.035-0.005-0.030>0, \\
L<\frac{0.04}{0.012 \cdot 2 \cdot 150} \approx 1 \cdot 10^{-2} \mathrm{H}, \\
\frac{30}{150}>2 \cdot 0.035 \rightarrow 0.2>0,07
\end{gathered}
$$

we are convinced that the conditions for stability of the arc welding mode selected by us, are performed with a «margin».

As the typical disturbing effects we use the step changes (jumps) of open-circuit voltage $\Delta u_{x}$ and the electrode feed speed $\Delta v_{e}$ :

$$
\begin{aligned}
& u_{x}(t)=\left\{\begin{array}{cc}
u_{x}, & t<t_{*}, \\
u_{x}+\Delta u_{x}, & t \geq t_{*},
\end{array}\right. \\
& v_{e}(t)=\left\{\begin{array}{cc}
v_{e}, & t<t_{*}, \\
v_{e}+\Delta v_{e}, & t \geq t_{*} .
\end{array}\right.
\end{aligned}
$$

Here $t_{*}$ is the moment of arising relevant effects.

The experiment results are presented in Figures 2 and 3. In these Figures, the solid lines depict the processes described by the specified mathematical model (3), and the dashed lines depict the processes described by the initial model (1).

The diagrams, presented in Figure 2, reflect the reaction of welding circuit on the voltage jump $\Delta u_{x}=$ $=3 \mathrm{~V}$. In this figure it is clearly seen, that at the moment of jump (at $t_{*}=3 \mathrm{~s}$ ) the welding current $i(t)$ and the electrode melting rate $v_{m}$ are almost instantly increased (the rate of increasing $i(t)$ and $v_{m}(t)$ is as higher, the lower is the ratio $L / R_{+}$). Whereas the arc voltage $u_{a}(t)$ is increased considerably slower, and as slower, the higher is the electric welding time constant $T_{w} \approx R_{w} /\left[K E\left(u_{x}-2 R_{+} i_{\infty}\right]\right.$. The estimated value $T_{w} \approx 0.085 \mathrm{~s}$. Then, the current $i(t)$ and rate $v_{m}(t)$ also decrease slowly. At the end of transition process (after the time $\tau \approx 3 T_{w}$ ), the variables $i(t)$ and $u_{a}(t)$ accept new values, moreover, $u_{a}(t)$ becomes higher and $i(t)$ lower as compared to their values $\Delta u_{x}$ before disturbance. Whereas the rate of melting $v_{m}(t)$ is preset as equal to the electrode feed speed $v_{e}$.

The diagrams, presented in Figure 3 reflect the reaction of the same variables $u_{a}(t), i(t)$ and $v_{m}(t)$ on the jump of electrode feed speed $\Delta v_{e}=5 \mathrm{~mm} / \mathrm{s}$. Here the situation is quite different. First of all, the change of all the variables occurs slowly (at the rate determined by the parameter $T_{w}$ ). At the end of the transition processes the new values of all the variables, including also $v_{m}(t)$ are established corresponding to the new electrode feed speed $v_{e}+\Delta v_{e}$. 

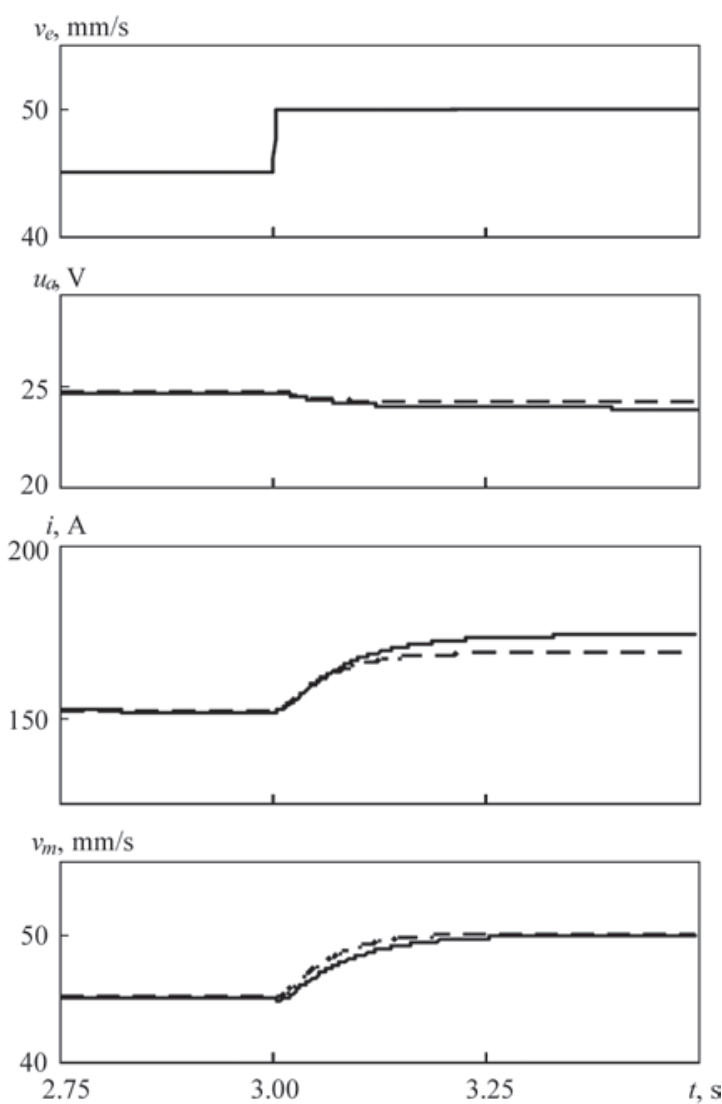

Figure 3. Reaction of welding circuit on disturbance $\Delta v_{e}=5 \mathrm{~mm} / \mathrm{s}$

A significant difference in the quickness of reaction of welding circuit to disturbances $\Delta u_{x}$ and $\Delta v_{e}$ is easy to understand, if to follow the propagation paths of these disturbances from the points of their application to the points of measuring variables $i(t), u_{a}(t)$, $v_{m}(t)$ (see schematic block diagram in Figure 4). Here it is appropriate to note that this difference is often overlooked, but meanwhile, its taking into account in the development of different methods of pulsed arc welding can turn to be decisive.

By comparing the transition processes depicted in Figures 2 and 3 in solid and dashed lines, we find that the reactions of welding current $i(t)$ one and on the same disturbance $\Delta u_{x}$ vary significantly. Namely this fact indicates that in the mathematical model (3), unlike in the model (1), the dependence of the electrode melting rate $v_{m}(t)$ not only on the actual value of welding current $i(t)$, but also on the actual value of arc voltage $u_{a}(t)$ is taken into account

In conclusion, we shall note that the results of the simulation experiment are well correlated with the experimental data presented in $[7,14]$ and demonstrate a fairly good compliance of the mathematical model (3) with the real dynamic processes, running in the welding circuit during arc consumable electrode welding.

\section{Conclusions}

1. The mathematical model (3), as compared to the model (1), reflects the essential aspects of dynamic pro-

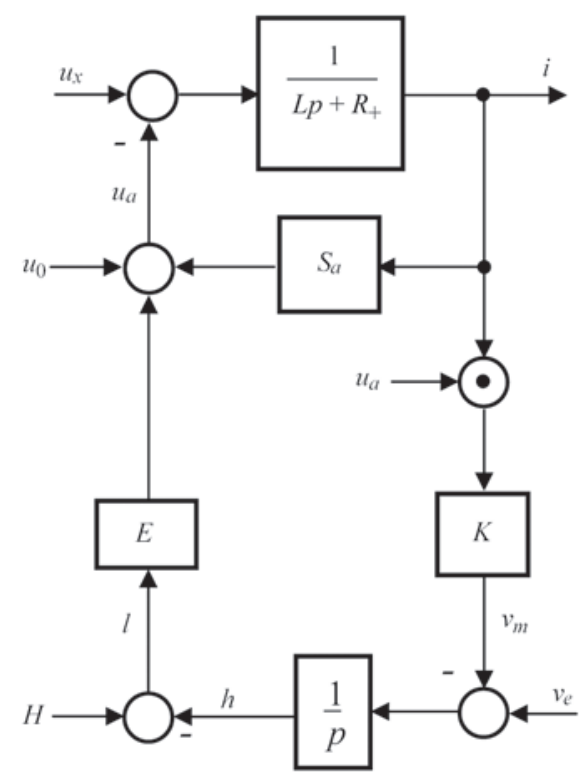

Figure 4. Schematic block diagram of welding circuit

cesses more precisely. Consequently, it provides an opportunity to extract also the more precise information about these processes, which is necessary both at the profound study of the considered processes, as well as in solving tasks of adaptive control of these processes.

2 . The criteria of stability (9), (12) and (13), established on the basis of the mathematical model (3), in addition to the already known criteria, impose certain limits on the parameters of welding arc mode, which is particularly valuable in practice.

1. Pan, J. (2003) Arc welding control. Woodhead Publishing Ltd.

2. Tsybulkin, G.A. (2014) Adaptive control in arc welding. Kiev: Stal.

3. Sugitani, Y. (2000) Making best use of the arc sensor. J. of JWS, 69(2), 46-50.

4. Ushio, M. (1991) Sensors in arc welding. Transact. of JWRI, 20(2), 157-163.

5. Sudnik, V.A., Ivanov, A.V. (1998) Mathematical model of heat source in metal-arc shielding-gas welding. Pt 1. Normal process. Svarochn. Proizvodstvo, 9, 3-9.

6. Korinets, I.F. (1995) Mathematical model of electrode wire melting in arc welding. Avtomatich. Svarka, 10, 39-43.

7. Paton, B.E., Lebedev, V.K. (1966) Electric equipment for arc and slag welding. Moscow: Mashinostroenie.

8. Leskov, G.I. (1970) Electric welding arc. Moscow: Mashinostroenie.

9. Barbashin, E.A. (1967) Introduction in stability theory. Moscow: Nauka.

10. Finkelnburg, V., Mekker, G. (1961) Electric arcs and thermal plasma. Moscow: Inostr. Literatura.

11. Nikitin, V.P. (1934) Electric machines and transformers for arc welding. Moscow; Leningrad: Energoizdat.

12. Tsybulkin, G.A. (2008) On the influence of small parameters on MIG/MAG welding stability. The Paton Welding J., 8, 22-25.

13. Dyurgerov, N.G., Sagirov, Kh.N. (2009) Stability of system of arc self-adjustment in mechanized and automatic welding. Svarochn. Proizvodstvo, 2, 13-14.

14. (1986) Automation of welding processes. Ed. by V.K. Lebedev et al. Kyiv: Vyshcha Shkola. 